\title{
Critical Role of Oxidant and Anti-oxidant in Cancer
}

\section{Kartick C Pramanik ${ }^{1}$ and Akhilesh Pandey ${ }^{2 *}$}

${ }^{1}$ Department of Biomedical Sciences and Cancer Biology Center, Texas Tech University of Health Sciences Center, Amarillo, Texas-79106, USA

${ }^{2}$ Garrison Institute on Aging, Department of Neurology, Texas Tech University Health Sciences Center, Lubbock, Texas- 79430, USA

Human life cannot survive without oxygen and at the same time it gives constant threat to the human being by producing toxic reaction. Oxidative stress is considered as a misbalance between free radicals and antioxidant levels and to be involved in the pathophysiology of all cancer. Many free radicals are unstable and highly reactive, such as hydroxyl radical $(\mathrm{OH})$, superoxide radical $\left(\mathrm{O}_{2}^{-}\right)$, nitric oxide radical (NO), Lipid peroxyl radical (LOO). It is noteworthy; free radicals are not always harmful. They also serve useful purposes in the human body as, it play role in the destruction of disease-causing microbes by specialized blood cells called phagocytes.

In normal cells, there is always an equal balance between reactive oxygen species (ROS) generation and anti-oxidant defense including glutathione peroxidase, superoxide dismutase, catalase which decreases the concentration of most harmful oxidants. Oxidative stress is generated by the disturbance of these pro-oxidants and anti-oxidants balance and it can damage all type of important cellular components, like proteins, carbohydrates, DNA and membrane lipids, and leads to cell death. Now the question is whether oxidative stress generated by external agent is beneficial for cancer cells. In recent years, several studies have revealed that oxidative stress generated by different agents is involved in a large number of pathological states, including carcinogenesis [1,2]. Studies have also shown that generation of reactive oxygen species by different natural and synthetic agents prevent cancer by inducing cancer cell death [3-5]. As a proof, 2-mothoxy estradiol is a new anticancer agent currently in clinical trials [6]. Several natural and synthetic drugs like capsaicin, chloroquin, quinine, mefloquine, primaquine, artemisinin, ciprofloxacin, are known to prevent the growth of cancer cells by generating reactive oxygen species [7-9].

Most of the cancer cells have higher levels of ROS that helps in proliferation and cell growth [10]. Excessive ROS generation by external agents increases threshold levels in cancer cells, and then they are highly dependent on their antioxidant system to maintain redox balance and hence are more susceptible to further oxidative stress. On the other hand, normal cells are more resistant to oxidative stress due to the fact that these cells have lower levels of ROS and increased levels of antioxidants. Recent study has shown that natural agent which increases intracellular ROS in cancer cells may increase ROS to a toxic level resulting in mitochondrial damage and cell death [9]. It is important to mention that several agents such as Elesclomol or Trisenx selectively kill cancer cells by increasing ROS generation are currently being used for the treatment of metastatic melanoma and acute promyelocytic leukemia respectively [11].

The main mechanism behind of increased production of reactive oxygen species by several external agents is to inhibition of mitochondrial electron chain complexes. As we all know, mitochondria are the main production house of reactive oxygen species which are generated due to incomplete reduction of oxygen during mitochondrial respiration. In normal conditions, mitochondria contain sufficient levels of antioxidants that prevent excess ROS generation and oxidative damage. However, in situations when ROS production is crossed it's threshold levels or when antioxidant levels are depleted, oxidative stress causes protein oxidation and lipid peroxidation, which damage mitochondrial membrane, proteins and DNA [12], leading to cancer cell death. Further, it induces apoptosis in human cancer cells, while inhibition of anti-oxidant enzyme, superoxide dismutase (SOD), causes accumulation of superoxide radicals in cancer cells that result in cell death [9]. Furthermore, it inhibits catalase and thereby increases oxidative stress and induces apoptosis in pancreatic cancer cells [9].

The Electron Transport Chain (ETC) complex in the mitochondria play major role in the generation of reactive oxygen species. In the process of breaking down carbon substrates into acetyl CoA, reducing equivalents (NADH and $\mathrm{FADH}$ ) are produced, which are then enter into the electron transport chain consisting of Complex I (NADH dehydrogenase), Complex II (succinate dehydrogenase), Complex III (ubiquinol cytochrome $c$ reductase), and Complex IV (cytochrome-c oxidase). After that, electrons move from the reducing equivalents to complex I and II, respectively, and then passed onto ubisemiquinone for shuttling to Complex III, followed by Complex IV through cytochrome-c. This electron shuttling process results in the development of a transmembrane proton electrochemical gradient. Complex-I and complex-III of the ETC are the major sites for ROS generation; some studies have targeted the ETC complex for induction of apoptosis and cancer treatment $[13,14]$.

From the above evidence, it was cleared that direct targeting of mitochondrial ETC by natural or synthetic agent increases ROS production from the mitochondria of cancer cells, which results in increased susceptibility to apoptotic induction. On the contrary, oxidative damage of any cellular constituent can contribute to disease development that may lead to cancer formation. Cancer cells develop an enhanced constitutive oxidative stress level, which sustains tumor growth, and shields these cells against pro-apoptotic signals, thus promoting tumor progression.

Therefore, it can been concluded that potential anti-cancer drugs act by either increasing reactive oxygen species or inhibiting endogenous anti-oxidant defense in order to selectively induce oxidative stress and apoptosis in cancer cells and suggests new therapeutic strategies that take advantage of increased ROS in cancer cells to enhance therapeutic activity and selectivity.

\section{References}

1. Harman D (1956) Aging: a theory based on free radical and radiation chemistry J Gerontol 11: 298-300.

2. Muller FL, Lustgarten MS, Jang Y, Richardson A, Van Remmen H (2007) Trends in oxidative aging theories. Free Radic Biol Med 43: 477-503.

3. Aggarwal BB, Shishodia S (2006) Molecular targets of dietary agents for prevention and therapy of cancer. Biochem Pharmacol 71: 1397-1421.

4. Buiatti E, Palli D, Bianchi S, Decarli A, Amadori D, et al. (1991) A case-contro

*Corresponding author: Akhilesh K. Pandey, Garrison Institute on Aging Department of Neurology, Texas Tech University Health Sciences Center, School of Medicine, Lubbock, Texas- 79430, USA, Tel: (806)743-1382; Fax: (806) 743-3636; E-mail: akhilesh.pandey@ttuhsc.edu

Received July 09, 2013; Accepted July 13, 2013; Published July 16, 2013

Citation: Pramanik KC, Pandey A (2013) Critical Role of Oxidant and Anti-oxidant in Cancer. Mol Biol 2: e110. doi:10.4172/2168-9547.1000e110

Copyright: (c) 2013 Pramanik KC, et al. This is an open-access article distributed under the terms of the Creative Commons Attribution License, which permits unrestricted use, distribution, and reproduction in any medium, provided the original author and source are credited. 
study of gastric cancer and diet in Italy. III. Risk patterns by histologic type. Int J Cancer 48: 369-374.

5. Pramanik KC, Srivastava SK (2012) Apoptosis signal-regulating kinase 1-thioredoxin complex dissociation by capsaicin causes pancreatic tumor growth suppression by inducing apoptosis. Antioxid Redox Signal 17: 14171432.

6. Ershler WB, Longo DL (1997) Aging and cancer: issues of basic and clinical science. J Natl Cancer Inst 89: 1489-1497.

7. Finkel T, Holbrook NJ (2000) Oxidants, oxidative stress and the biology of ageing. Nature 408: 239-247.

8. Raha S, Robinson BH (2000) Mitochondria, oxygen free radicals, disease and ageing. Trends Biochem Sci 25: 502-508.

9. Pramanik KC, Boreddy SR, Srivastava SK (2011) Role of mitochondrial electron transport chain complexes in capsaicin mediated oxidative stress leading to apoptosis in pancreatic cancer cells. PLoS One 6: e20151.
10. Trachootham D, Zhou Y, Zhang H, Demizu Y, Chen Z, et al. (2006) Selective killing of oncogenically transformed cells through a ROS-mediated mechanism by beta-phenylethyl isothiocyanate. Cancer Cell 10: 241-252.

11. Toogood PL (2008) Mitochondrial drugs. Curr Opin Chem Biol 12: 457-463.

12. Hamanaka RB, Chandel NS (2010) Mitochondrial reactive oxygen species regulate cellular signaling and dictate biological outcomes. Trends Biochem Sci 35: 505-513.

13. Chung WG, Miranda CL, Maier CS (2007) Epigallocatechin gallate (EGCG potentiates the cytotoxicity of rotenone in neuroblastoma SH-SY5Y cells. Brain Res 1176: 133-142.

14. Dong LF, Low P, Dyason JC, Wang XF, Prochazka L, et al. (2008) Alphatocopheryl succinate induces apoptosis by targeting ubiquinone-binding sites in mitochondrial respiratory complex II. Oncogene 27: 4324-4335 